\title{
A Nano-Platform for in situ Investigations of Ovarian Follicles
}

Jean M. Feugang ( $\square$ j.feugang@msstate.edu )

Mississippi State University https://orcid.org/0000-0002-1376-5059

Ghassan Ishak

Southern Illinois University Carbondale

Matthew Eggert

Auburn University

Robert Arnold

Auburn University

Scott Willard

Mississippi State University

Peter Ryan

Mississippi State University

Eduardo Gastal

Southern Illinois University Carbondale

\section{Research}

Keywords: Liposome vesicles, Molecular imaging, Nanoparticles, Targeted drug delivery, Ovarian follicles, Intrafollicular injection, Livestock, Assisted reproductive technology

Posted Date: September 22nd, 2020

DOI: https://doi.org/10.21203/rs.3.rs-78063/v1

License: (c) (i) This work is licensed under a Creative Commons Attribution 4.0 International License.

Read Full License 


\section{A nano-platform for in situ investigations of ovarian follicles}

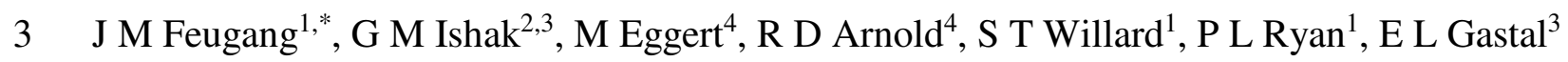
4

$5{ }^{1}$ Department of Animal and Dairy Sciences, Mississippi State University, Mississippi State, MS,

$6 \quad U S A$

$7 \quad{ }^{2}$ Department of Surgery and Obstetrics, College of Veterinary Medicine, University of Baghdad,

8 Baghdad, Iraq

$9{ }^{3}$ Department of Animal Science, Food and Nutrition, Southern Illinois University, Carbondale,

10 Illinois, USA

$11{ }^{4}$ Department of Drug Discovery and Development, Harrison School of Pharmacy, Auburn

12 University, Auburn, AL, USA

13

14

$15 *$ Corresponding author: Jean M. Feugang, Department of Animal and Dairy Sciences,

16 Mississippi State University, Mississippi State, MS, USA. 335 Wise Center, PO Box 9815

17 Mississippi State, MS 39762. Tel. (662) 325-7567; FAX: (662) 325-8873 E-mail:

18 j.feugang@msstate.edu

19

20 


\section{Abstract}

22 Background: Despite the growing array of assisted reproductive techniques, there is still a lack

23 of rapid, non-cytotoxic, and minimally invasive in situ approaches for further enhancements

24 through cell targeting. Here we synthesized clinically relevant liposome nanoparticles for real-

25 time cellular targeting and drug (doxorubicin) delivery, using pigs and mares as animal models.

26 In Experiment 1, fluorescently labeled and doxorubicin-loaded (without fluorescent probe)

27 liposomes were injected in cultured pig ovarian follicles to assess plasma membrane binding and

28 intracellular doxorubicin delivery. In Experiment 2, fluorescent liposomes were in vivo injected

29 into small and large ovarian follicles of living mares to assess their binding capability to

30 follicular cells. Twenty-four hours post-injection, all cultured pig follicles were collected while

31 mare samples (i.e., follicle wall fragments, granulosa cells and follicular fluids) were harvested

32 through follicle wall biopsy (FWB), and follicle aspiration and flushing techniques using

33 transvaginal ultrasound-guided approach.

34 Results: All injected follicles were healthy and samples were subjected to fluorescence imaging

35 before and after fixation. Findings revealed successful intrafollicular migration and binding of

36 liposomes to all follicle cell layers (granulosa, theca interna, and theca externa) regardless of the

37 follicle size. The intracellular delivery of doxorubicin was confirmed with the staining of nuclei

38 of follicle cells.

39 Conclusions: This study demonstrates the promising combination of the FWB technique and

40 nanotechnology tools for real-time monitoring of intrafollicular treatment, follicular health and

41 oocyte development, which in turn has the potential to help understand the mechanisms of

42 ovulatory dysfunction and to select high-quatity oocytes for assisted reproduction techniques. 
44 Keywords: Liposome vesicles, Molecular imaging, Nanoparticles, Targeted drug delivery,

45 Ovarian follicles, Intrafollicular injection, Livestock, Assisted reproductive technology

\section{Background}

48 The current efforts to overcome fertility problems through assisted reproductive technologies

49 (ARTs) are still unsatisfactory because of the insufficient maturation of the oocytes and/or the

50 existing follicular diseases [1-6]. Furthermore, the ability to assess the quality of the oocytes

51 mainly, if not only, through in vitro manipulations constitutes a serious limitation [7, 8]. Hence,

52 the development of novel methods for in situ assessment of oocyte quality and local (versus

53 systemic) treatments of unhealthy follicles prior to the retrieval of enclosed oocytes would be of

54 great interest.

55 Challenges related to female fertility treatments need the investigation of various

56 methodologies. For example, the routine systemic drug injections (i.e., exogenous

57 gonadotropins) for controlled ovarian hyperstimulation $(\mathrm{COH})$ is showing increased evidence of

58 detrimental effects on oogenesis, embryo quality, and endometrial receptivity [9-12]. Hence,

59 intrafollicular injection, which has been applied in large animals (e.g., cow and mare) with

60 relative success $[4,13]$, may become a viable alternative to $\mathrm{COH}$, and the recent advances in

61 nanotechnology and assisted reproduction tools are likely to boost the outcomes of intrafollicular

62 treatments.

63 At present, the primary use of "naked" biomolecules, without a carrier, for intrafollicular

64 injection has drastically limited their potential to gain mechanistic insights related to follicle 
65 growth and oocyte maturation. Interestingly, the recent detection of extracellular membrane

66 microvesicles in equine [14], human [15], and bovine [16] follicular fluid (FF) and their roles as

67 delivery carriers of biomolecules (e.g., lipids, proteins, RNAs, and miRNA) enabling

68 intercellular communications have prompted new interest in the use of liposome nanoparticles

69 for intrafollicular injections [17-19]. Liposomes are clinically relevant carbon-based

70 nanoparticles shaped like spherical vesicles constituted of phospholipid bilayers providing

71 biocompatibility, biodegradability, and low toxicity to cells [20]. These nanoparticles have been

72 used as effective carriers for controlled and rapid deliveries of various hydrophilic and

73 hydrophobic molecules to specific biological sites, with indubitable benefits during in vivo and in

74 vitro applications [21, 22]. For fertility treatments, several drugs, including biomolecules that are

75 found in the FF [23-27], can be loaded into liposomes for intrafollicular injections.

76 Following intrafollicular injections, the minimally invasive in vivo Follicle Wall Biopsy

77 (FWB) technique is a recently improved methodology for assisted reproduction allowing for

78 simultaneous sampling of the follicle environment (FF, granulosa, theca interna and externa

79 cells) for ex vivo examinations. Most importantly, the FWB technique is performed on living

80 mares without affecting their fertility, ovarian function, or the FF composition, therefore

81 providing an excellent cocktail for biomarkers research and clinical purposes [28, 29]. Its

82 combination with the encapsulated liposomes for intrafollicular deliveries can be highly effective

83 for a more in-depth understanding of the molecular mechanisms governing follicle growth and

84 oocyte maturation.

85 In the current study, fluorescent and non-fluorescent unilamellar liposome vesicle

86 nanoparticles were synthesized. Fluorescence was used for the visualization of the tissular 
87 distribution, and non-fluorescent liposomes were loaded with doxorubicin to assess intercellular

88 delivery. The effectiveness of the liposome nanoparticles in binding the plasma membrane of

89 follicle cells and delivering doxorubicin was tested in vitro with cultured pig ovarian follicles (ex

90 situ - Experiment 1). Thereafter, as the first step to implement in situ fertility treatments and

91 deep-tissue imaging, the binding capability of liposomes in living animals was tested using intact

92 equine ovarian follicles (in situ - Experiment 2).

\section{Results}

\section{Characterization of synthesized liposome nanoparticles}

96 The formulated liposome characterization in Tris buffer $\mathrm{pH} 7.5$ indicated a z-average

97 nanoparticle diameter of $124.7 \mathrm{~nm}(\mathrm{PDI}=0.106)$, a number average diameter of $87.7 \pm 27.5 \mathrm{~nm}$

98 (Fig. 1A), and a zeta potential mean of $-22.9 \pm 9.51 \mathrm{mV}$ (Fig. 1B). The addition of 1\% DiI to the

99 formulated fluorescent liposomes led to a final hydrodynamic average diameter of $109.9 \pm 17.5$

$100 \mathrm{~nm}$, while the TEM imaging indicated a dry average diameter of $21.3 \pm 4.5 \mathrm{~nm}( \pm \mathrm{SEM})$,

101 calculated for thirty random liposomes (Fig. 1A- Insert). For this study, three independent

102 liposomes were formulated with high fluorescence intensity $\left(1.9 \times 10^{11} \pm 8 . \times 10^{9}\right.$ radiance; Fig.

103 2A). Their mixture and dilution with PBS resulted in the working liposome solution of $23.1 \mu \mathrm{M}$

104 DiI dye with fluorescence intensity of $6.5 \times 10^{10}$ radiance (IVIS imaging; Fig. 2B).

105

107 porcine follicles

108 IVIS imaging of dissected porcine antral follicles 
109 Decreasing fluorescence signals with PBS-injected liposomes (auto fluorescence; upper follicle

110 series) when compared to those injected with fluorescence liposomes (middle follicle series) or

111 liposomal encapsulated doxorubicin (lower follicle series) can be seen in Figure 3A. Data

112 quantification confirmed higher $(\mathrm{P}<0.05)$ fluorescence signals of both liposome- $\left(1.35 \times 10^{9} \pm\right.$

$\left.1135.31 \times 10^{8}\right)$ and doxorubicin-injected follicles $\left(1.82 \times 10^{9} \pm 8.38 \times 10^{8}\right)$ and radiant efficiency,

114 when compared with the PBS-injected group $\left(3.93 \times 10^{8} \pm 1.53 \times 10^{8}\right.$; Figure $\left.3 \mathrm{~B}\right)$; however, the

115 fluorescence signals in both liposome and doxorubicin groups were comparable $(\mathrm{P}>0.05)$.

\section{Microscopy imaging of liposome binding and doxorubicin delivery}

118 Imaging was performed on follicle wall sections on microscope slides. Autofluorescence or

119 low/background fluorescence can be observed in sections of follicles injected with PBS (Fig.

120 4A). In contrast, the highest fluorescence intensity observed in the plasma membranes of follicle

121 wall cells confirmed the liposome binding (Fig. 4B). The successful binding of doxorubicin-

122 loaded liposomes to cellular plasma membranes and the delivery of doxorubicin $(10 \mu \mathrm{g} /$ follicle $)$

123 to the nucleus (red dots) of various cell types of the follicle wall (Fig. 4C) were observed; and

124 highlights the colocalization of red (doxorubicin) and blue staining (DAPI) within the nuclei can 125 be seen in Figure 4D- Insert.

127 Experiment 2. In vivo liposome binding in living mares and ex vivo imaging

128 A total of 22 growing and healthy follicles of nine mares (2-3 follicles/mare) were successfully

129 injected with $130 \mu \mathrm{l}$ of fluorescent liposomes working stock solution. 


\section{IVIS imaging of FWB}

132 All FWB samples were of comparable areas $\left(3.5 \pm 0.3 \mathrm{~mm}^{2}\right)$, and those of liposome-injected

133 follicles exhibited fluorescence intensities above background (Control; $6.7 \times 10^{8} \pm 1.44 \times 10^{7}$

134 radiant efficiency; Fig. 5A). The FWB derived from both small and large follicles had

135 comparable fluorescence intensities $\left(1.74 \times 10^{9} \pm 7.6 \times 10^{8}\right.$ and $1.98 \times 10^{9} \pm 3.4 \times 10^{8}$ radiant

136 efficiency, respectively, $\mathrm{P}>0.05$; Fig. 5B). In vitro labeling of FWB with an equivalent amount

137 of liposomes $\left(130 \mu \mathrm{l}=1.2\right.$ nmoles; $60 \mathrm{~min}$ at $\left.37^{\circ} \mathrm{C}\right)$ led to higher $\left(3.92 \times 10^{9} \pm 1.11 \times 10^{8}\right.$ radiant

138 efficiency, $\mathrm{P}<10^{-4}$ ) fluorescence intensity than that of other groups (Fig. 5B).

140 IVIS imaging of intrafollicular fluid

141 The FF of both non-injected (control) and PBS-injected follicles emitted low fluorescence

142 intensities that were considered background and subtracted from those of FF harvested from

143 small (Fig. 6A) and large (Fig. 6B) follicles injected with liposomes ( $<10^{-4}$; Fig. 6C). Data

144 quantification shows higher fluorescence intensity from small follicles’ FF $\left(2.95 \times 10^{9} \pm 9.65\right.$

$145 \times 10^{8}$ radiant efficiency) than their large counterparts $\left(8.53 \times 10^{8} \pm 0.98 \times 10^{8}\right.$ radiant efficiency).

147 IVIS imaging of granulosa cells (GC)

148 Fluorescence signals of GC harvested from both small and large follicles were above background

149 (non-injected or control follicles: $7.4 \times 10^{8} \pm 1.23 \times 10^{7}$ radiant efficiency, $\mathrm{P}<10^{-5}$; Fig. 7A).

150 Large follicles' GC produced higher fluorescence than that of small follicles $\left(1.61 \times 10^{9} \pm 6.83\right.$

$151 \times 10^{7}$ vs. $9.12 \times 10^{8} \pm 1.67 \times 10^{7}$ radiant efficiency, $\mathrm{P}<0.05$; Fig. 7B) 


\section{Confocal microscope fluorescence imaging of FWB samples and flushed granulosa cells}

154 The uniform staining of cell nuclei with DAPI (blue) revealed the good health status of harvested

155 samples. Follicles injected with PBS did not show any fluorescence (green staining)

156 corresponding to the presence of liposomes (Fig. 8A). In contrast, follicles injected with

157 liposomes displayed green fluorescence staining within the plasma membrane of the follicle wall

158 cells (Fig. 8B). The insert in Figure 8B highlights the fluorescence signal on the plasma

159 membrane surrounding the blue staining of the nuclei; this observation is confirmed with the

160 images of flushed mural granulosa cells showing stronger fluorescence signals within the plasma

161 membrane (Fig. 8C,D). The smeared fluorescence signals seen in Figure 8C,D on the tissues

162 surrounding the GC correspond to liposomes bound/trapped within the extracellular matrix.

\section{Discussion}

165 Many factors, such as the physiological conditions of animals and the available technologies, are

166 prone to influence assisted reproduction outcomes in animals. Here we examined a clinically

167 relevant liposome vesicle nanoparticle as a potentially powerful, non-invasive diagnostic and

168 therapeutic agent for future assessment and treatment of ovarian follicles prior to oocyte

169 recovery. Monovular (mare) and polyovular (pig) species were used to demonstrate the likely

170 application of our proposed technological approach for in situ follicle investigation and

171 improvement of female fertility.

172 Herein, the sizes of the synthesized liposomes were comparable to those in previous reports

$173[20,30,31]$, while the injected volumes ( $5 \mu 1$ in pigs and $200 \mu 1$ in mares $)$ and incubation time

174 (24 h) were lesser or equal to those reported in previous studies [4, 32]. Hence, the absence of 
175 morphological changes in both equine and porcine ovarian follicles' structures following

176 intrafollicular injections was highly important, with the prospect of performing local deliveries

177 (i.e., intrauterine or intrafollicular) of molecules at minimal effective doses to reach the targeted

178 cells during ovarian follicle treatments [33]. These minimal effective doses following local

179 injections (vs. systemic treatments) were demonstrated in mares [34] and cows [13] using PGF2 $\alpha$

180 ( $\leq 0.1 \mathrm{mg}$ vs. up to 50-fold greater) and hCG (1.0 IU vs. up to 1000-fold greater), respectively.

181 The ability to by-pass the systemic route (i.e., intramuscular or intravenous) and avoid the

182 dilution with the blood-stream through local injections of growing antral follicles permits the

183 study of the direct effects of biocompounds (insulin-like growth factor 1, eCG, and PGF2 $\alpha$ )

184 during folliculogenesis $[4,34,35]$. These studies highlight the promising interest in

185 intrafollicular treatments in animal production and veterinary medicine; however, most related

186 studies use "naked" (devoid of additional carriers) biomolecules whose ability to cross cell

187 membranes and short lifespan may be jeopardized during long incubations or journeys.

188 Interestingly, the current progress in nanotechnology is increasing the popularity of

189 nanoparticles in biomedicine. These nanoparticles possess specific properties (e.g., size-

190 dependent synthesis, bright and broad-spectrum fluorescence, biocompatibility and

191 bioconjugation capability) that can be exploited for further investigation and improvement of

192 reproductive outcomes $[22,32,36]$. In the present study, we used liposomes shaped like

193 extracellular membrane microvesicles (EVs), which are nano- and micro-size vesicles naturally

194 released from cells to mediate a new type of cell-to-cell communication through passive

195 interactions $[17,18,37]$. Likewise, liposomes can interact and fuse with somatic cells (e.g.,

196 MCF-7 breast cancer cells) within approximately five minutes post-incubation [38]. In the 
197 present study, the pig model allowed the (IVIS) imaging of the full and intact follicle, indicating

198 the possibility for in situ non-invasive monitoring of folliculogenesis through molecular imaging

199 of key biological processes. With the existing fluorescence spectrum of hydrophobic dyes, it is

200 reasonable to anticipate the formulation of specific labeled liposomes for multiplex molecular

201 imaging for real-time examinations of various follicular phenomena related to aging, ovarian

202 cyclicity (cycling vs. anestrous phase), structural variations (antral follicles vs. corpus luteum),

203 reproductive phases (estrous $v s$. diestrous), and health (follicle diseases). Understanding of them

204 may have beneficial implications for women, who are known to share many reproductive

205 similarities with mares [39].

206 The present study highlights the advantage of combining nanotechnology with granulosa cell

207 aspiration and in vivo FWB techniques to provide more insights into the intrafollicular

208 microenvironment. Recently, the FWB technique was validated for simultaneous collections of

209 follicle wall fragments and FF samples from the same living mare [28, 29]. Here, we first

210 observed that the expected unidirectionality of liposome binding, from the follicular antrum to

211 the theca externa, leading to lower fluorescence intensity of in situ labeled biopsies contrasted

212 with the likely multidirectional binding during ex situ labeling; consequently, all follicle cell

213 types simultaneously interacted with the liposomes ex situ to generate stronger fluorescence

214 signals. Second, the higher fluorescence of FFs in small follicles was indicative of the low

215 abundance of cells capable of uptaking liposomes. And third, the high abundance of cells in large

216 follicles permitted the binding of more liposomes, leading to reduced unbound fluorescent

217 liposomes in the corresponding FFs. Nonetheless, fluorescence intensity variations between 
218 samples certainly corresponded to the possible losses of liposomes during injections, while the

219 higher fluorescence of large follicles' granulosa cells was attributed to their greater density.

220 The random and passive characteristics of injected liposomes to fuse with follicular cells'

221 membranes makes them suitable for intracellular deliveries. Indeed, liposomes are among the

222 numerous carriers with efficient intracellular delivery of cargo of molecules such as proteins,

223 nucleic acids, metabolites, and even drugs [14, 17, 18, 36, 38]. Our findings revealed the

224 effective internalization of doxorubicin with its binding to the cell nucleus of all follicle cell

225 types, demonstrating the far-reaching capacity of the proposed injection route.

226

227 Conclusions

228 The current study demonstrates 1) the combination of FWB technique and nanotechnology tools

229 as a promising approach for potential local treatments of ovarian follicle diseases and

230 enhancement of reproductive performance; 2) the possibility for deep-tissue (plain or targeted)

231 imaging using fluorescent-labeled liposomes for in situ tracking of molecular and/or cellular

232 processes throughout the antral follicle growth; and 3) the possible use of a formulated liposome

233 carrier in both mono- and polyovulator animals as well as in living large animals. We believe the

234 proposed methodology is likely to support future intrafollicular attempts to promote follicle

235 and/or oocyte growth and to develop treatments to address follicle diseases such as luteinized

236 unruptured follicle in mares and, potentially, in women as well $[5,40]$.

238 Methods

239 Chemical and reagents 
240 Phospholipid, DPPC (1, 2-dipalmitoyl-sn-glycero-3-phosphocholine) was purchased from Avanti

241 Polar Lipids, Inc. (Alabaster, Alabama, USA). Ultrapure grade cholesterol was obtained from

242 Amresco, Inc. (Solon, Ohio, USA). Lipophilic probes, 1, 1'-dioctadecyl-3, 3, 3', 3'-

243 tetramethylindocarbocyanine perchlorate (DiIC18(3) or DiI) and 3,3'-

244 dioctadecyloxacarbocyanine perchlorate (DiOC18(3) or DiO), were acquired from Biotium, Inc.

245 (Hayward, California, USA). Any additional reagents, chemicals, or solvents of necessary

246 molecular biology or analytical grade were obtained through commercial sources.

\section{Liposome nanoparticle preparation and characterization}

249 Fluorescence liposome formulation. Pegylated liposomes were prepared as previously described

$250[41,42]$. Briefly, formulations were generated according to a ratio of $10 \mu \mathrm{mol}$ DPPC, $5 \mu \mathrm{mol}$

251 cholesterol, and $100 \mathrm{nmol}$ of the fluorescent lipophilic probe, DiI ( 1 mol \% of lipid membrane),

252 per milliliter of dispersant. The lipids and probe, dissolved in chloroform, were mixed and then

253 dried under a rotary vacuum via a Buchi Rotavapor r-200 (Postlfach, Switzerland) to create a

254 thin film. Subsequently, films were hydrated in an isotonic solution of either 10\% (w/v) sucrose

255 or phosphate-buffered saline to yield $10 \mu \mathrm{mol}$ phospholipid/mL. Liposomes underwent seven

256 freeze/thaw cycles via liquid nitrogen and a $60^{\circ} \mathrm{C}$ water-bath above lipid phase transition

257 temperature as a means to eliminate additional membrane layers. Formulations were then

258 extruded using a Lipex extruder (Northern Lipids Inc., Burnaby, British Columbia, Canada)

259 under high pressure through double-stacked laser etched polycarbonate $80 \mathrm{~nm}$ filters (GE

260 Osmonics, Trevose, Pennsylvania, USA) at heated conditions again above DPPC's phase

261 transition temperature $\left(\mathrm{T}_{\mathrm{c}}=41^{\circ} \mathrm{C}\right)$ for seven times before stabilizing the lipid membranes using 
262 an ice-bath for $10 \mathrm{~min}$. Afterward, the liposome dispersions were dialyzed with a $12-14 \mathrm{kDa}$

263 SpectraPor Dialysis membranes against fresh isotonic solution as used in hydration for a period

264 of 1,4 , and 16 hours at $4^{\circ} \mathrm{C}$ to remove any non-membrane associated probe. Liposomes were

265 sterilized by terminal filtration $(0.22 \mu \mathrm{m}$ filter $)$ and stored in a sterile glass container under

266 nitrogen atmosphere at $4^{\circ} \mathrm{C}$, protected from light, and used within 3 weeks of preparation. Lipid

267 concentration was determined for all formulations using an inorganic phosphate assay [43].

268 Thereafter, the dispersion was diluted to $10 \mu \mathrm{mol} / \mathrm{ml}(10 \mathrm{mM})$, and average stock solutions of

269 liposome nanoparticles $(9.24 \pm 0.72 \mu \mathrm{mol} / \mathrm{ml}$ or $9.24 \mathrm{mM})$ were constituted and stored at $4^{\circ} \mathrm{C}$ for

270 experiments. The DiI lipophilic probe fluoresced at $570 \mathrm{~nm}$ following a $480 \mathrm{~nm}$ wavelength

271 excitation.

272

273 Doxorubicin-loaded liposome formulation. A nonfluorescent pegylated liposomal was

274 formulated by Avanti Polar Lipids, Inc. (Alabaster, Alabama, USA) and used to encapsulate the 275 naturally fluorescing doxorubicin (Dox-NP; referenced maxima Ex/Em of 480nm/590nm). All

276 samples were stored at $4^{\circ} \mathrm{C}$ for experiments.

277

278 High-Resolution Transmission Electron Microscopy and Fluorescence imaging. A subset of

279 synthesized liposomes was visualized under a high-resolution transmission electron microscope 280 (HR-TEM-2100, 200 kV) with Gatan Orius 832 camera (JEOL; Peabody, Massachusetts, USA), 281 while another subset was subjected to non-invasive and real-time fluorescence imaging using the 282 In Vivo Imaging System 200, Lumina XRMS Series III system (IVIS; PerkinElmer; Waltham, 283 Massachusetts, USA). 
285 Experiment 1. Porcine antral follicle culture and intrafollicular liposome injection

286 Porcine ovaries were harvested in a pre-warmed $0.9 \% \mathrm{NaCl}$ solution at a local slaughterhouse

287 and transported immediately to the laboratory. Visible and morphologically normal antral

288 follicles (4-8 $\mathrm{mm}$ in diameter) were dissected and randomly distributed in a set of 24-well tissue

289 culture plates (3-4 follicles/well); subsequently, the tissue culture plates were placed in a 5\%

$290 \mathrm{CO}_{2}$ incubator as previously reported [32]. After $24 \mathrm{~h}$ of culture at $37^{\circ} \mathrm{C}$ in a humidified

291 environment, follicles were microinjected using FemtoJet microinjection system (Eppendorf;

292 Hauppauge, New York, USA) with $5 \mu$ l of Phosphate-Buffered Saline solution (PBS) or 46 nmol

293 of fluorescent liposomes. Thereafter, injected follicles were returned to their respective culture

294 wells with half of the medium renewed (1.5 $\mathrm{ml}$ of NCSU-23 based medium) and re-incubated for

295 an additional day of culture. A subset of non-injected follicles was kept in culture to serve as

296 controls for auto-fluorescence. Following culture, follicles were carefully removed from culture

297 wells and washed twice in PBS solution prior to non-invasive IVIS fluorescence imaging.

298 Thereafter, follicles were processed for standard histology and fluorescence microscopic

299 imaging.

300

301 Experiment 2. In vivo liposome binding tests in living mares

302 Animals, ultrasonography examination, and intrafollicular fluorescent liposome injection.

303 Nine nonlactating and cycling Quarter Horse breed mares, 8-14 years old, and weighing

$304400-600 \mathrm{Kg}$ were used during the ovulatory season. Mares were kept under natural light in

305 pasture and were maintained with free access to water and trace-mineralized salt. Ovarian follicle 
306 tracking was performed daily starting on day 10 of the estrous cycle (day $0=$ ovulation) until

307 follicles reached the designated diameter. Ultrasonographic follicle tracking was carried out

308 using a duplex color Doppler ultrasound machine (Aloka SSD-3500; Hitachi Aloka Medical

309 America, Inc., Wallingford, CT, USA) equipped with a finger-mounted 3.5-10 MHz convex-

310 array transducer (UST-995-7.5). After identification and mapping of small $(15-23 \mathrm{~mm} ; \mathrm{n}=12)$

311 and large $(24-30 \mathrm{~mm} ; \mathrm{n}=10)$ follicles that showed continuous growth for a minimum of three

312 consecutive days, fluorescent liposome nanoparticles $(130 \mu \mathrm{l} ; 23.1 \mu \mathrm{M}$ or $1.2 \mathrm{nmol})$ were

313 injected into each growing follicle using a double-channel injection system as previously

314 described $[4,44]$. All mares $(n=9)$ were used for the injection of 22 follicles (12 small and 10

315 large), corresponding to 2-3 follicles per mare. One small and one large noninjected follicle

316 served as experimental controls. After follicle injections, all mares were released to the pasture

317 for $24 \mathrm{~h}$. Thereafter, all samples (FWB, FF, and isolated granulosa cells) were collected for 318 analyses.

320 Mare antral follicle wall biopsy and follicular fluid collection. A novel FWB technique recently

321 developed in our lab [29] was used to simultaneously harvest FWB and FF from PBS- and

322 liposome-injected follicles (7 small and 7 large) and one non-injected (control) follicle. This

323 technique uses an endoscopic biopsy forceps (5 FR gauge, $60 \mathrm{~cm}$, Karl Storz, Berlin, Germany)

324 located inside a $12 \mathrm{G}$ needle/cannula and mounted on a 5-10 MHz transvaginal ultrasound-

325 guided convex array transducer (Aloka UST-987-7.5). The FWB samples measured

326 approximately $3.5 \pm 0.3 \mathrm{~mm}^{2}$ in area, as previously reported [29]. Immediately after FWB

327 harvesting, FF was collected while the needle was still inside the follicle. Intrafollicular fluid 
328 samples were centrifuged at $4^{\circ} \mathrm{C}(1,500 \mathrm{~g}$ for $10 \mathrm{~min})$, and the supernatant of each FF sample

329 was stored in light safe black Eppendorf tubes at $4^{\circ} \mathrm{C}$.

331 In situ collection of equine granulosa cells. The remaining injected (6 small and 5 large) and

332 non-injected (1) follicles were flushed with $120-150 \mathrm{ml}$ of pre-warmed $\left(38^{\circ} \mathrm{C}\right)$ Vigro complete

333 embryo flush with BSA solution (Bioniche; Pullman, WA, USA) containing $2 \mathrm{IU} / \mathrm{ml}$ heparin

334 (H3149, Sigma-Aldrich; Saint-Louis, MO, USA), and granulosa cells were retrieved. Thereafter,

335 flushing fluid was filtered through a $70-\mu \mathrm{m}$ cell strainer, and concentrated granulosa cells were

336 washed out from the strainer with PBS into a petri dish and transferred into light safe black

337 Eppendorf tubes. After centrifugation ( $800 \mathrm{~g}$ for $10 \mathrm{~min}$ ) at $4^{\circ} \mathrm{C}$, the supernatant was discarded,

338 and the pelleted granulosa cells were kept at $4^{\circ} \mathrm{C}$ until fluorescence imaging.

\section{$340 \quad$ Fluorescence microscopic imaging}

341 All samples were imaged within a week post-collection.

343 IVIS imaging. Equine FF, granulosa cells, and FWB samples, as well as cultured porcine

344 follicles, were collected and subjected to non-invasive and real-time fluorescence imaging using

345 the IVIS imaging system (PerkinElmer; Waltham, Massachusetts, USA).

347 Fluorescence imaging. Following IVIS imaging, porcine follicles and equine FWB samples

348 were fixed in $10 \%$ formalin solution for standard histology processing, generating 4-6 $\mu \mathrm{m}$

349 sample sections per slide. Fixed equine granulosa cells were spread on histology slides, air-dried, 
350 immersed with a DAPI-contained mounting medium, and covered by a cover-slip. Slides were

351 imaged with an epifluorescence microscope (EVOS FL-Auto Cell Imaging System, Thermo

352 Fisher Scientific Inc., Waltham, MA, USA) or a confocal laser scanning with an Inverted

353 microscope (Axiovert 200M) coupled with Zeiss ZEN digital imaging software (Carl Zeiss AG;

354 Oberkochen, Germany).

355

356 Statistical analyses

357 All statistical analyses were performed using SPSS v22 software. The Student's t-test and One358 way Analysis of Variance (ANOVA) were used, wherever appropriate, to compare the difference 359 between groups (control, small and large follicles). The threshold of significance was set for p360 values less or equal to 0.05 . All data are expressed as mean \pm SEM.

362 List of abbreviations

363 PBS: Phosphate-buffered saline; FWB: Follicle Wall Biopsy; DAPI: 4',6-diamidino-2-

364 phenylindole; GC: Granulosa Cells; FF: Follicular Fluid; IVIS: In Vivo Imaging System; DiI:

365 1,1'-Dioctadecyl-3,3,3',3'-Tetramethylindocarbocyanine Perchlorate; SPSS: Statistical Product 366 and Service Solutions.

368 Ethics approval and consent to participate

369 All experimental procedures were performed according to the United States Government

370 Principles for the Utilization and Care of Vertebrate Animals Used in Testing, Research and

371 Training (grants.nih.gov/grants/olaw/references/phspol.htm). The use of animals and procedures 
372 were approved by the Institutional Animal Care and Use Committee of Southern Illinois

373 University.

374

375 Consent for publication

376 Not applicable.

377

378 Availability of data and materials

379 Data sharing is not applicable to this article as no datasets were generated or analyzed during the 380 current study.

382 Competing interests

383 The authors declare that they have no competing interests.

385 Funding

386 This material is based upon work that is supported by the National Institute of Food and

387 Agriculture, U.S. Department of Agriculture, Hatch project accession number 1016077 and 388 Multistate number W4171. Additional funds for research and APC were provided by the USDA389 ARS Biophotonics (58-6402-3-018).

390

391 Authors' contributions

392 JMF and ELG conceptualized the study; ME and RDA synthesized and characterized the 393 liposome nanoparticles; JMF, GMI, and ELG performed the experiments and collected data; 
394 JMF, GMI, ME, and ELG analyzed the data and wrote the first draft of the manuscript; RDA,

395 PLR and STW contributed to the conceptualization and data interpretation. All authors read and

396 approved the final manuscript.

398 Acknowlegdments

399 The authors thank Dr. Melba Gastal and Gabriel Dutra for their technical assistance.

\section{References}

1. Motlik J, Crozet N, Fulka J: Meiotic competence in vitro of pig oocytes isolated from early antral follicles. Reproduction 1984, 72:323-328.

2. Gilchrist RB, Thompson JG: Oocyte maturation: emerging concepts and technologies to

improve developmental potential in vitro. Theriogenology 2007, 67:6-15.

3. Feugang JM, Camargo-RodrÃ-guez O, Memili E: Culture systems for bovine embryos. Livestock Science 2009, 121:141-149.

4. Gastal EL, Kot K, Ginther OJ: Ultrasound-guided intrafollicular treatment in mares.

Theriogenology 1995, 44:1027-1037.

5. Bashir S, Gastal MO, Tazawa SP, Tarso S, Hales DB, Cuervo-Arango J, Baerwald AR, Gastal EL: The mare as a model for luteinized unruptured follicle syndrome: intrafollicular endocrine milieu. Reproduction 2016, 151:271-283.

6. Hinrichs K: Production of embryos by assisted reproduction in the horse. Theriogenology 1998, 49:13-21.

7. Lonergan P, Fair T: Maturation of Oocytes in Vitro. Annual Review of Animal Biosciences 2016, 4:255-268.

8. Hansen PJ: Implications of Assisted Reproductive Technologies for Pregnancy Outcomes in Mammals. Annual Review of Animal Biosciences 2020, 8:395-413.

9. Horcajadas JA, Mínguez P, Dopazo J, Esteban FJ, Domínguez F, Giudice LC, Pellicer A, Simon C: Controlled ovarian stimulation induces a functional genomic delay of the endometrium with potential clinical implications. The Journal of Clinical Endocrinology \& Metabolism 2008, 93:4500-4510. 
10. Haouzi D, Assou S, Dechanet C, Anahory T, Dechaud H, De Vos J, Hamamah S: Controlled ovarian hyperstimulation for in vitro fertilization alters endometrial receptivity in humans: protocol effects. Biology of reproduction 2010, 82:679-686.

11. Lu C-L, Yan Z-Q, Song X-L, Xu Y-Y, Zheng X-Y, Li R, Liu P, Feng H-L, Qiao J: Effect of exogenous gonadotropin on the transcriptome of human granulosa cells and follicular fluid hormone profiles. Reproductive Biology and Endocrinology 2019, 17:49.

12. Sharma V, Whitehead M, Mason B, Pryse-Davies J, Ryder T, Dowsett M, Campbell S, Collins W: Influence of superovulation on endometrial and embryonic development. Fertility and Sterility 1990, 53:822-829.

13. Kot K, Gibbons J, Ginther O: A technique for intrafollicular injection in cattle: effects of hCG. Theriogenology 1995, 44:41-50.

14. da Silveira JC, Veeramachaneni DR, Winger QA, Carnevale EM, Bouma GJ: Cell-secreted vesicles in equine ovarian follicular fluid contain miRNAs and proteins: a possible new form of cell communication within the ovarian follicle. Biology of reproduction 2012, 86:71, 71-10.

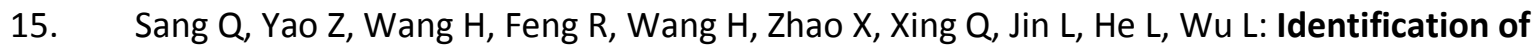
microRNAs in human follicular fluid: characterization of microRNAs that govern steroidogenesis in vitro and are associated with polycystic ovary syndrome in vivo. The Journal of Clinical Endocrinology \& Metabolism 2013, 98:3068-3079.

16. Hailay T, Hoelker M, Poirier M, Gebremedhn S, Rings F, Saeed-Zidane M, Salilew-Wondim D, Dauben C, Tholen E, Neuhoff C: Extracellular vesicle-coupled miRNA profiles in follicular fluid of cows with divergent post-calving metabolic status. Scientific reports 2019, 9:1-14.

17. Di Pietro C: Exosome-mediated communication in the ovarian follicle. Journal of assisted reproduction and genetics 2016, 33:303-311.

18. Tesfaye D, Hailay T, Salilew-Wondim D, Hoelker M, Bitseha S, Gebremedhn S: Extracellular vesicle mediated molecular signaling in ovarian follicle: Implication for oocyte developmental competence. Theriogenology 2020.

19. Dumesic DA, Meldrum DR, Katz-Jaffe MG, Krisher RL, Schoolcraft WB: Oocyte environment: follicular fluid and cumulus cells are critical for oocyte health. Fertility and sterility 2015, 103:303-316.

20. Akbarzadeh A, Rezaei-Sadabady R, Davaran S, Woo Joo S, Zarghami N, Hanifehpour Y, Samiei M, Kouhi M, Nejati-Koshki K: Liposome: Classification, preparation, and Applications. Nanoscale Research Letters 2013, 8:102.

21. Grijalva M, Vallejo-López MJ, Salazar L, Camacho J, Kumar B: Cytotoxic and antiproliferative effects of nanomaterials on cancer cell lines: a review. Unraveling the Safety Profile of Nanoscale Particles and Materials: From Biomedical to Environmental Applications 2018:63.

22. Feugang JM, Rhoads CE, Mustapha PA, Tardif S, Parrish JJ, Willard ST, Ryan PL: Treatment of boar sperm with nanoparticles for improved fertility. Theriogenology 2019, 137:75-81.

23. Ferrazza RdA, Garcia HDM, Schmidt EMdS, Mihm Carmichael M, Souza FFd, Burchmore R, Sartori $\mathrm{R}$, Eckersall PD, Ferreira JCP: Quantitative proteomic profiling of bovine follicular fluid during follicle development. Biology of reproduction 2017, 97:835-849.

24. Fahiminiya S, Labas V, Roche S, Dacheux J-L, Gérard N: Proteomic analysis of mare follicular fluid during late follicle development. Proteome science 2011, 9:54.

25. Dutra G, Ishak G, Pechanova O, Pechan T, Peterson D, Jacob J, Willard S, Ryan P, Gastal E, Feugang J: Seasonal variation in equine follicular fluid proteome. Reproductive Biology and Endocrinology 2019, 17:29. 
26. Paes VM, Liao SF, Figueiredo JR, Willard ST, Ryan PL, Feugang JM: Proteome changes of porcine follicular fluid during follicle development. Journal of animal science and biotechnology 2019, 10:94.

27. Walter J, Huwiler F, Fortes C, Grossmann J, Roschitzki B, Hu J, Naegeli H, Laczko E, Bleul U: Analysis of the equine "cumulome" reveals major metabolic aberrations after maturation in vitro. BMC genomics 2019, 20:588.

28. Ishak GM, Dutra GA, Gastal GD, Gastal MO, Feugang JM, Gastal EL: Transition to the ovulatory season in mares: An investigation of antral follicle receptor gene expression in vivo. Molecular Reproduction and Development 2019, 86:1832-1845.

29. Ishak G, Bashir S, Dutra G, Gastal G, Gastal M, Cavinder C, Feugang J, Gastal E: In vivo antral follicle wall biopsy: a new research technique to study ovarian function at the cellular and molecular levels. Reproductive Biology and Endocrinology 2018, 16:71.

30. Wang $Q$, He L, Fan D, Liang W, Fang J: Improving the anti-inflammatory efficacy of dexamethasone in the treatment of rheumatoid arthritis with polymerized stealth liposomes as a delivery vehicle. Journal of Materials Chemistry $B$ 2020, 8:1841-1851.

31. Ren H, He Y, Liang J, Cheng Z, Zhang M, Zhu Y, Hong C, Qin J, Xu X, Wang J: Role of Liposome Size, Surface Charge, and PEGylation on Rheumatoid Arthritis Targeting Therapy. ACS Applied Materials \& Interfaces 2019, 11:20304-20315.

32. Feugang JM, Youngblood RC, Greene JM, Willard ST, Ryan PL: Self-illuminating quantum dots for non-invasive bioluminescence imaging of mammalian gametes. Journal of nanobiotechnology 2015, 13:38.

33. Ginther OJ, Siddiqui MAR, Beg MA: Progesterone responses to intravenous and intrauterine infusions of prostaglandin F2 in mares. Reproduction, Fertility and Development 2009, 21:688695.

34. Weber J, Causey R, Emmans E: Induction of luteolysis in mares by ultrasound-guided intraluteal treatment with PGF2alpha. Theriogenology 2001, 55:1769-1776.

35. Ginther O, Gastal E, Gastal M, Bergfelt D, Baerwald A, Pierson R: Comparative study of the dynamics of follicular waves in mares and women. Biology of reproduction 2004, 71:1195-1201.

36. Jha RK, Jha PK, Chaudhury K, Rana SVS, Guha SK: An emerging interface between life science and nanotechnology: present status and prospects of reproductive healthcare aided by nanobiotechnology. Nano Reviews 2014, 5:10.3402/nano.v3405.22762.

37. Yáñez-Mó M, Siljander PR-M, Andreu Z, Bedina Zavec A, Borràs FE, Buzas El, Buzas K, Casal E, Cappello F, Carvalho J: Biological properties of extracellular vesicles and their physiological functions. Journal of extracellular vesicles 2015, 4:27066.

38. Stathopoulos G, Boulikas T: Lipoplatin formulation review article. Journal of drug delivery 2012, 2012.

39. Carnevale $\mathrm{E}$ : The mare model for follicular maturation and reproductive aging in the woman. Theriogenology 2008, 69:23-30.

40. Bashir ST, Baerwald AR, Gastal MO, Pierson RA, Gastal EL: Follicle growth and endocrine dynamics in women with spontaneous luteinized unruptured follicles versus ovulation. Human Reproduction 2018, 33:1130-1140.

41. Quach N, Mock J, Scholpa N, Eggert M, Payre C, Lambeau G, Arnold R, Cummings B: Role of the phospholipase $\mathbf{A} 2$ receptor in liposome drug delivery in prostate cancer cells. Molecular pharmaceutics 2014, 11:3443-3451. 
515 42. Kamps JA, Morselt HW, Swart PJ, Meijer DK, Scherphof GL: Massive targeting of liposomes, surface-modified with anionized albumins, to hepatic endothelial cells. Proceedings of the National Academy of Sciences 1997, 94:11681-11685.

43. Bartlett GR: Phosphorus assay in column chromatography. Journal of Biological Chemistry 1959, 234:466-468. 


\section{Figure legends}

Figure 1. Liposome characterization through Malvern Zetasizer Nano ZS.

The Z-average diameter $(\mathbf{A})$ and Zeta potential $(\mathbf{B})$ parameters were measured in 3 replicates (dilutions 1,2, and 3). The insert is a TEM micrograph of liposome nanoparticles in monomer (arrows) and aggregate formations.

Figure 2. Fluorescence imaging of liposome formulations using In Vivo Imaging System (IVIS). Fluorescence imaging ( $480 \mathrm{~nm}$ excitation/570 nm emission) of 3 stock solutions (an average of $1.9 \times 10^{11} \pm 8 . \times 10^{9}$ radiance efficiency; $\left.\mathbf{A}\right)$ and the working solution $(\mathbf{B})$ are shown. The blue box surrounding the right panel (B) has a Phosphate-Buffered Saline solution (PBS) solution and diluted liposome solution $\left(23.1 \mu \mathrm{M}\right.$; an average of $6.5 \times 10^{10} \pm 8 . \times 10^{9}$ radiance $)$.

Figure 3. Fluorescence imaging of in vitro culture porcine follicles.

The micrograph (A) corresponds to fluorescence detected with In Vivo Imaging System (IVIS) of pig follicles microinjected with $5 \mu \mathrm{l}$ of Phosphate-Buffered Saline solution (PBS; upper series), fluorescence liposomes (4.6 nmoles/follicle; middle series), and liposomal encapsulated doxorubicin (10 $\mu \mathrm{g} /$ follicle; lower series) and cultured $24 \mathrm{~h}$ before imaging. All fluorescence quantifications are shown in $(\mathbf{B})$.

Figure 4. In situ fluorescence imaging of microinjected porcine follicles.

Following microinjection and $24 \mathrm{~h}$ culture of porcine follicles, follicles were fixed and subjected to standard histology processing. Representative micrographs with sections of antral follicle walls/layers injected with PBS (A, autofluorescence) or fluorescent liposomes ( $\mathbf{B}$, green color) are shown. The green fluorescence is mainly located on the cell plasma membrane.

Representative micrographs with sections of antral follicles injected with non-fluorescent liposomes loaded with doxorubicin are mainly located in the nuclei of follicle wall cells $(\mathbf{C}, \mathbf{D})$, which colocalization with DAPI (blue) is highlighted with the insert in Micrograph D, corresponding to fluorescence overlaid with visible light. Granulosa cells (GC), Theca interna (TI), and Theca externa (TE) constitute the cell layers of the ovarian follicle wall.

Figure 5. Representative fluorescence imaging of equine follicle wall biopsies (FWB). Biopsies were obtained from in vivo microinjected small (SF: $n=6)$ and large (LF: $n=5)$ follicles. In parallel, FWB samples were harvested from Phosphate-Buffered Saline solution (PBS)-injected follicles to serve as Control with a subset being in vitro labeled with comparable fluorescence liposome concentrations. All FWB samples were imaged with the In Vivo Imaging System (IVIS) and data (Radiant Efficiency) were quantified (mean \pm sem). Letters indicate significant differences $\left(\mathrm{a}, \mathrm{b}, \mathrm{c} ; \mathrm{P}<10^{-4}\right)$. $\mathrm{N}=$ total number of samples analyzed. 
565 Figure 6. Representative fluorescence imaging of equine follicular fluid (FF) following in vivo 566 labeling with fluorescence liposome nanoparticles.

567 The In Vivo Imaging System (IVIS) of FF collected from small and large follicles microinjected

568 with fluorescent liposomes is shown in $\mathbf{A}$ and $\mathbf{B}$, respectively. The quantification of collected

569 fluorescence signals is plotted minus the values of FF originated from Phosphate-Buffered Saline

570 solution (PBS)-microinjected follicles that were considered as the background noise.

571 Fluorescence signal (Radiant Efficiency) data are mean ( \pm SEM) and letters indicate a significant

572 difference $\left(\mathrm{a}, \mathrm{b} ; \mathrm{P}<10^{-4}\right) . \mathrm{N}=$ total number of samples analyzed.

Figure 7. Representative fluorescence imaging of in vivo labeled equine granulosa cells (GC). The micrograph (left panel) shows tubes containing GC collected from follicles injected with Phosphate-Buffered Saline solution (PBS) to serve as Control, and liposome-microinjected follicles (A). Mean ( \pm SEM) fluorescence data (Radiant Efficiency) of small (SF) and large (LF) follicles are indicated $(\mathbf{B})$. Letters denote significant differences $\left(\mathrm{a}, \mathrm{b}, \mathrm{c} ; \mathrm{P}<\mathrm{P}<10^{-5}\right)$. $\mathrm{N}=$ total 579 number of samples analyzed.

Figure 8. In vivo fluorescence imaging of liposome binding to equine ovarian antral follicles. Micrograph A showed a fragment of the follicle wall biopsy (FWB) of an antral follicle injected with Phosphate-Buffered Saline solution (PBS). A representative sample of FWB harvested from

584 antral follicles injected with fluorescent liposomes are shown in Micrograph B. Granulosa cells 585 flushed from liposome injected antral follicles are shown in Micrographs $\mathbf{C}$ and $\mathbf{D}$. The green 586 fluorescence detection of liposome was detected in the mural granulosa cells layers and the major signal was located in the cells' plasma membrane (Insert in micrograph B), which is also

588 confirmed in Micrographs $\mathbf{C}$ and D. Nuclei are counterstained in blue with DAPI. 


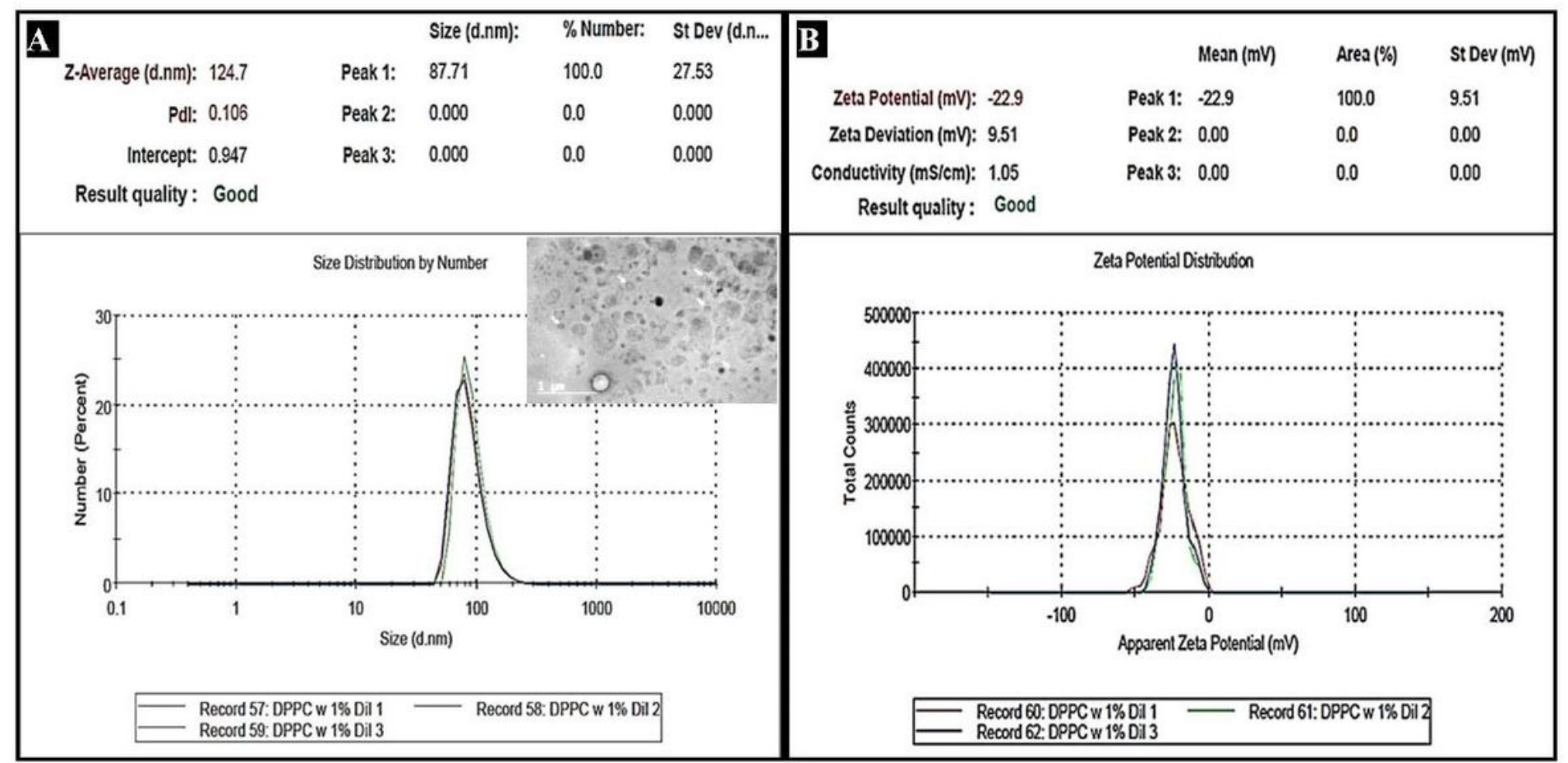

\section{Figure 1}

Liposome characterization through Malvern Zetasizer Nano ZS. The Z-average diameter (A) and Zeta potential (B) parameters were measured in 3 replicates (dilutions 1,2, and 3 ). The insert is a TEM micrograph of liposome nanoparticles in monomer (arrows) and aggregate formations.
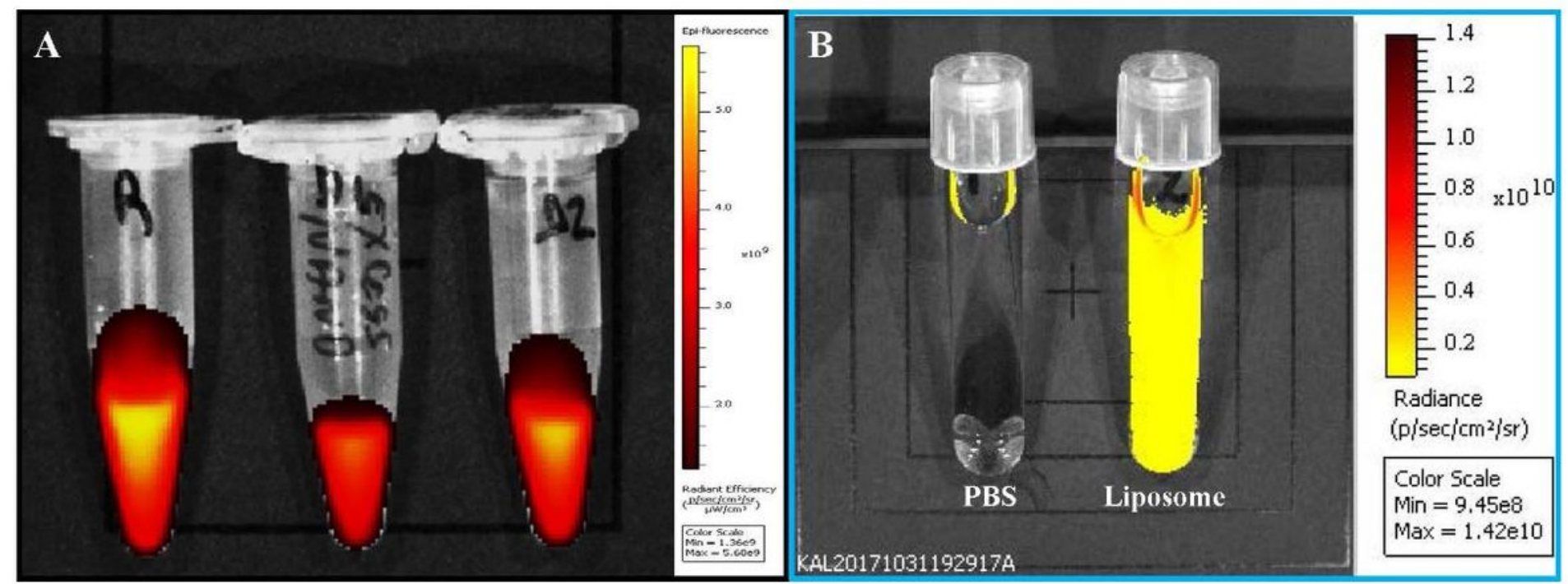

Figure 2 
Fluorescence imaging of liposome formulations using In Vivo Imaging System (IVIS). Fluorescence imaging (480 nm excitation/570 nm emission) of 3 stock solutions (an average of 1.9x1011 $\pm 8 . \times 109$ radiance efficiency; $A$ ) and the working solution (B) are shown. The blue box surrounding the right panel (B) has a Phosphate-Buffered Saline solution (PBS) solution and diluted liposome solution (23.1 $\mu \mathrm{M}$; an average of $6.5 \times 1010 \pm 8 . \times 109$ radiance).
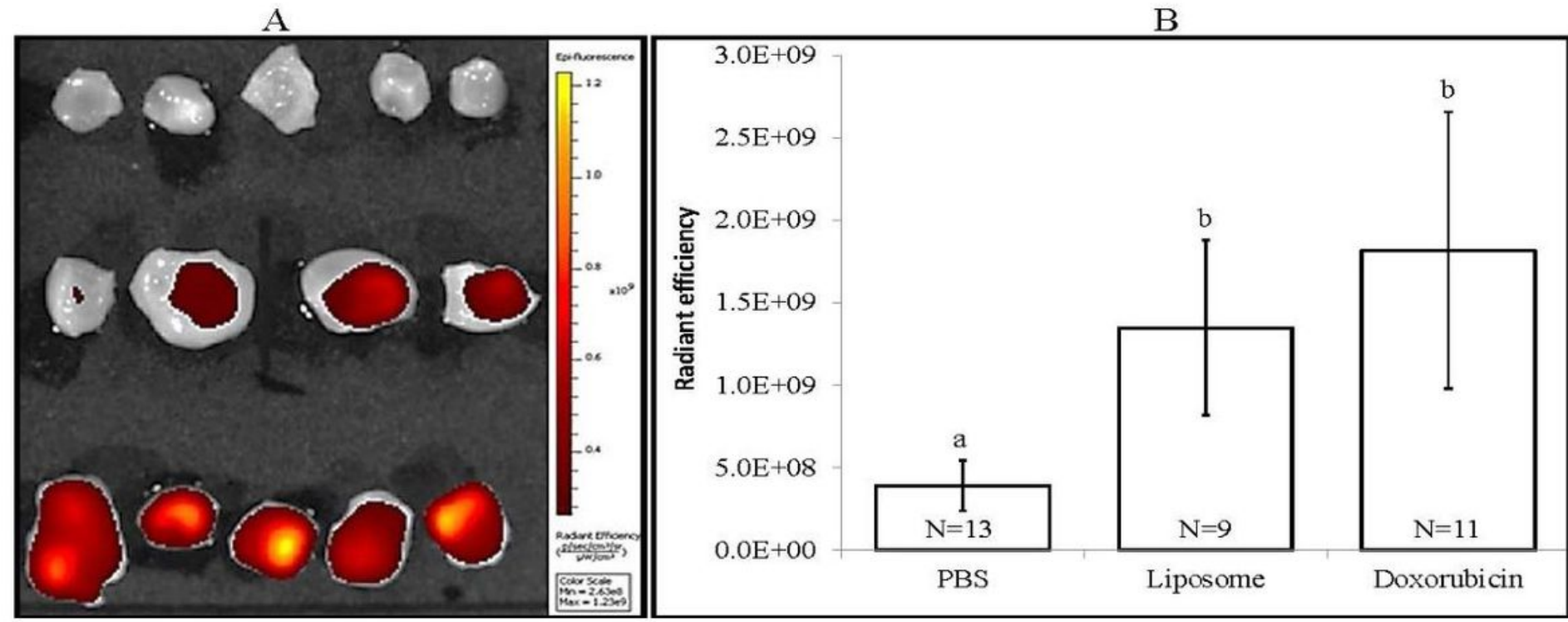

\section{Figure 3}

Fluorescence imaging of in vitro culture porcine follicles. The micrograph (A) corresponds to fluorescence detected with In Vivo Imaging System (IVIS) of pig follicles microinjected with $5 \mu$ l of Phosphate-Buffered Saline solution (PBS; upper series), fluorescence liposomes (4.6 nmoles/follicle; middle series), and liposomal encapsulated doxorubicin (10 $\mathrm{\mu g}$ /follicle; lower series) and cultured $24 \mathrm{~h}$ before imaging. All fluorescence quantifications are shown in (B). 



\section{Figure 4}

In situ fluorescence imaging of microinjected porcine follicles. Following microinjection and $24 \mathrm{~h}$ culture of porcine follicles, follicles were fixed and subjected to standard histology processing. Representative micrographs with sections of antral follicle walls/layers injected with PBS (A, autofluorescence) or fluorescent liposomes (B, green color) are shown. The green fluorescence is mainly located on the cell plasma membrane. Representative micrographs with sections of antral follicles injected with nonfluorescent liposomes loaded with doxorubicin are mainly located in the nuclei of follicle wall cells (C, D), which colocalization with DAPI (blue) is highlighted with the insert in Micrograph D, corresponding to fluorescence overlaid with visible light. Granulosa cells (GC), Theca interna (TI), and Theca externa (TE) constitute the cell layers of the ovarian follicle wall. 

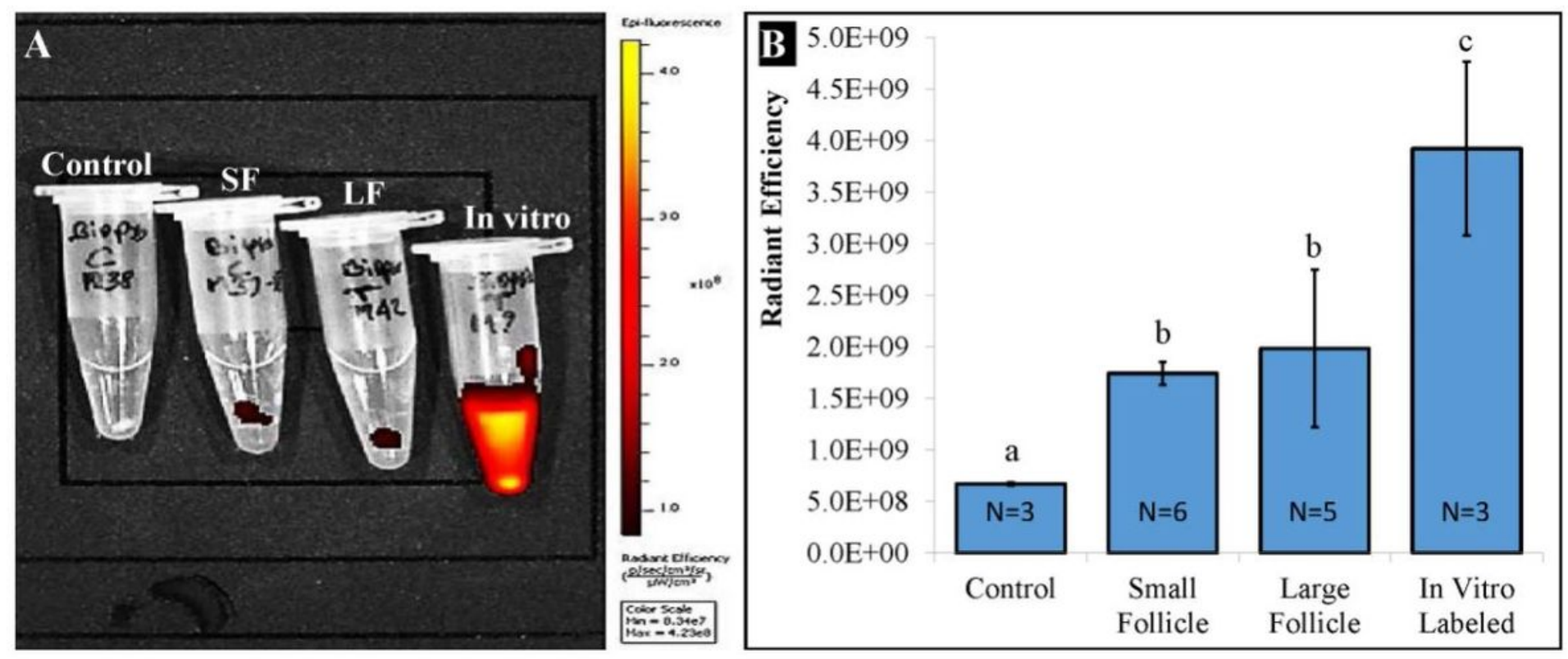

Figure 5

Representative fluorescence imaging of equine follicle wall biopsies (FWB). Biopsies were obtained from in vivo microinjected small (SF: $n=6)$ and large (LF: $n=5)$ follicles. In parallel, FWB samples were harvested from Phosphate-Buffered Saline solution (PBS)-injected follicles to serve as Control with a subset being in vitro labeled with comparable fluorescence liposome concentrations. All FWB samples were imaged with the In Vivo Imaging System (IVIS) and data (Radiant Efficiency) were quantified (mean \pm sem). Letters indicate significant differences $(a, b, c ; P<10-4) . N=$ total number of samples analyzed.
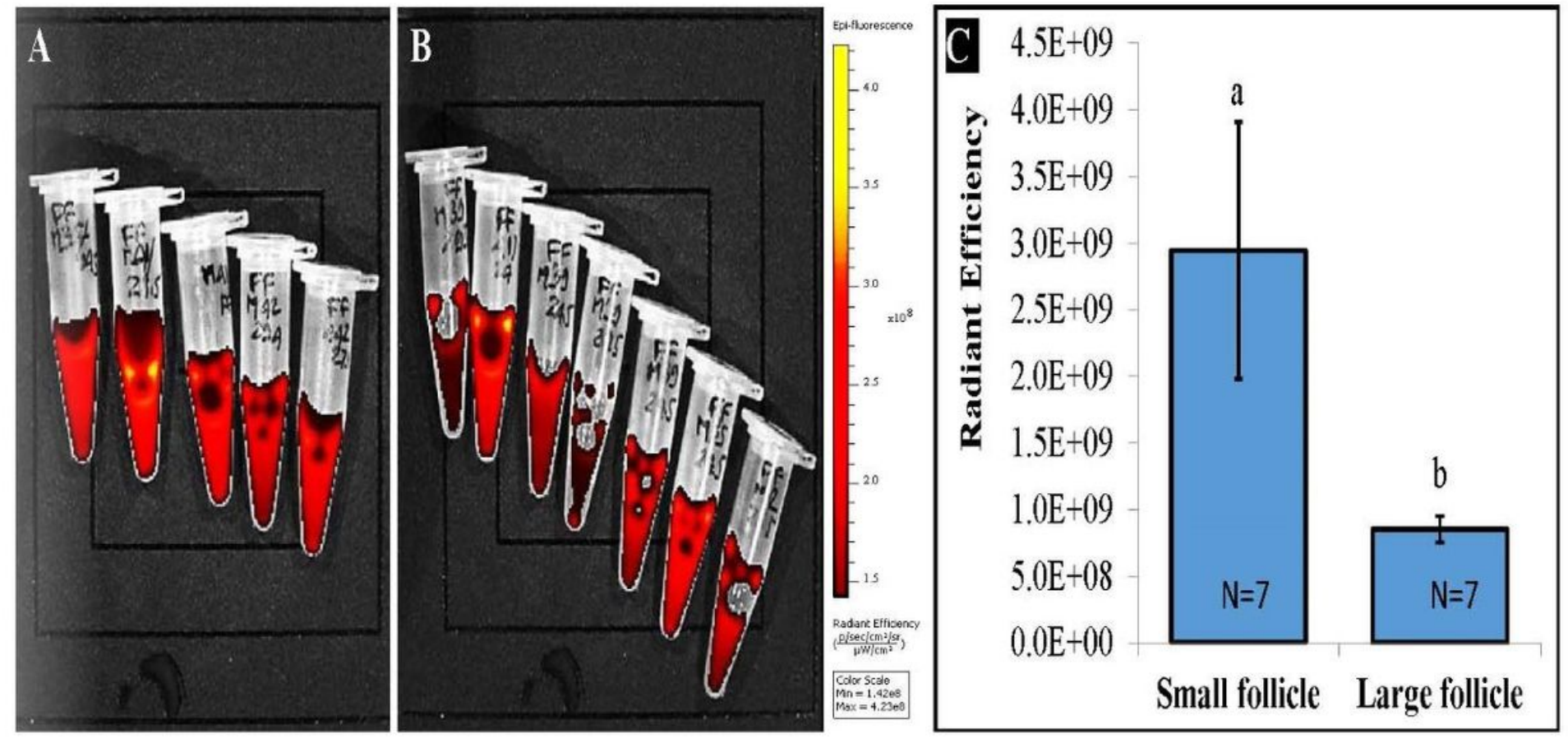

Figure 6 
Representative fluorescence imaging of equine follicular fluid (FF) following in vivo labeling with fluorescence liposome nanoparticles. The In Vivo Imaging System (IVIS) of FF collected from small and large follicles microinjected with fluorescent liposomes is shown in A and B, respectively. The quantification of collected fluorescence signals is plotted minus the values of FF originated from Phosphate-Buffered Saline solution (PBS)-microinjected follicles that were considered as the background noise. Fluorescence signal (Radiant Efficiency) data are mean ( \pm SEM) and letters indicate a significant difference $(a, b ; P<10-4) . N=$ total number of samples analyzed.
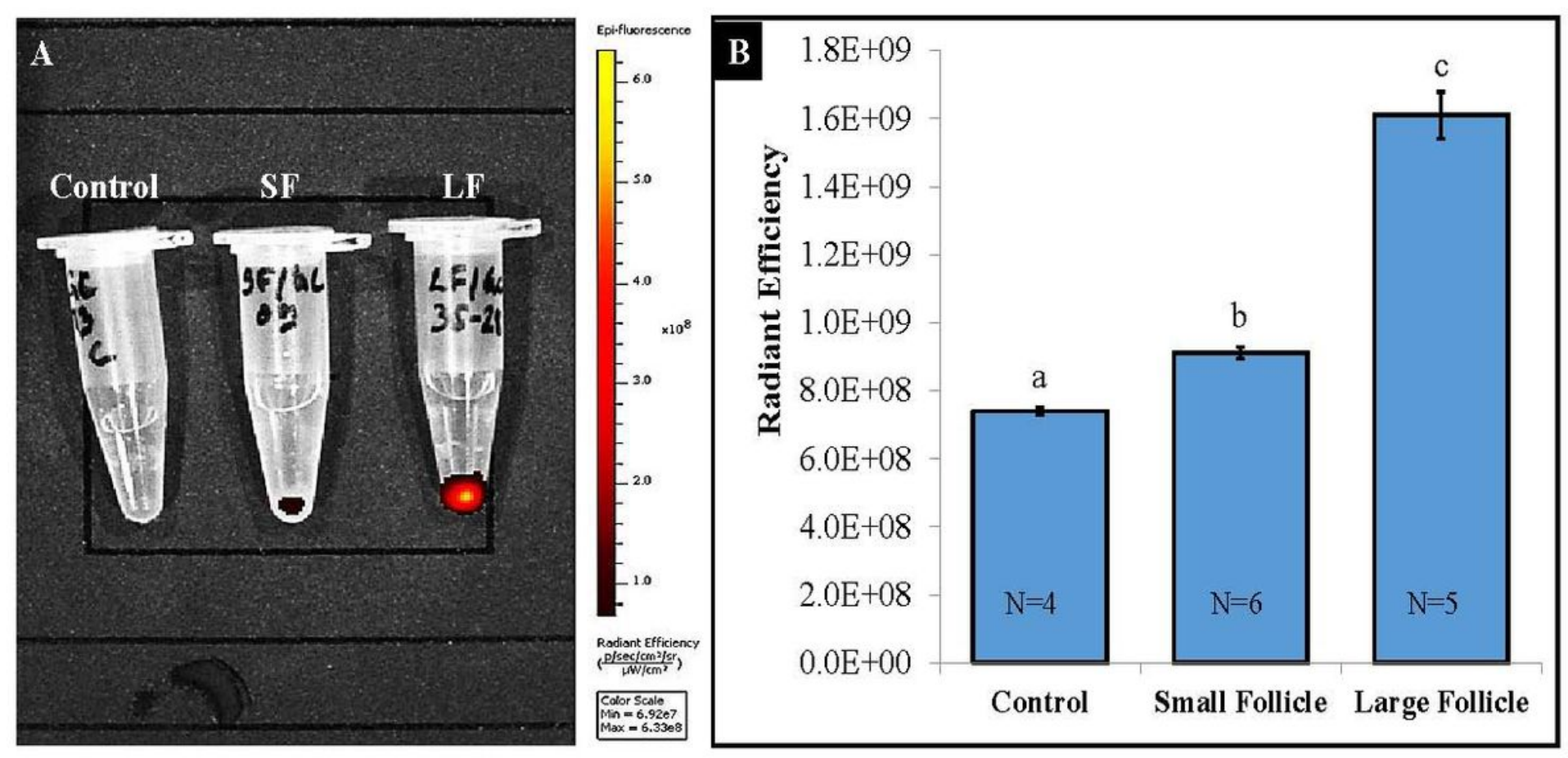

Figure 7

Representative fluorescence imaging of in vivo labeled equine granulosa cells (GC). The micrograph (left panel) shows tubes containing GC collected from follicles injected with Phosphate-Buffered Saline solution (PBS) to serve as Control, and liposome-microinjected follicles (A). Mean ( \pm SEM) fluorescence data (Radiant Efficiency) of small (SF) and large (LF) follicles are indicated (B). Letters denote significant differences $(a, b, c ; P<P<10-5) . N=$ total number of samples analyzed. 

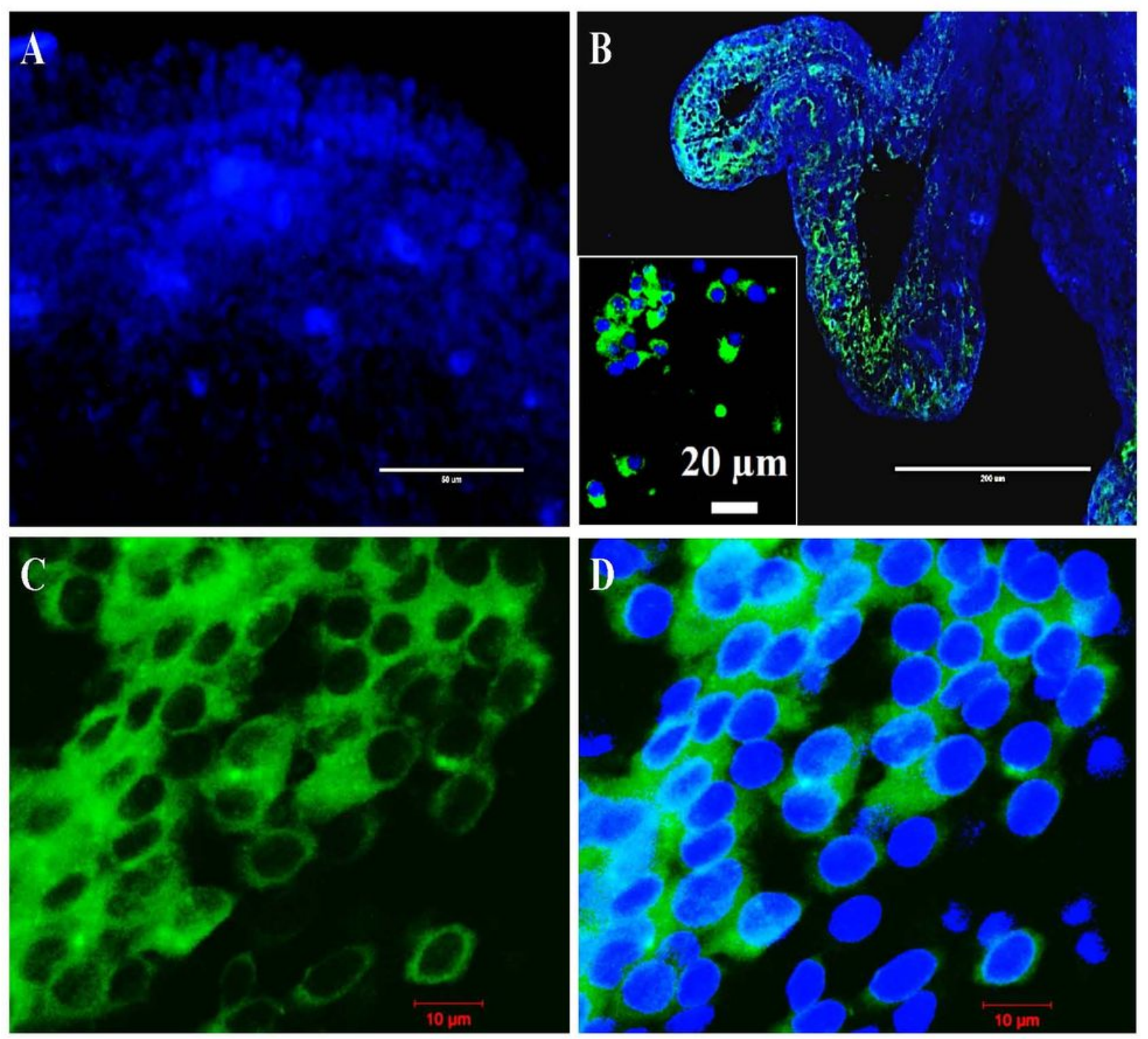

\section{Figure 8}

In vivo fluorescence imaging of liposome binding to equine ovarian antral follicles. Micrograph A showed a fragment of the follicle wall biopsy (FWB) of an antral follicle injected with Phosphate-Buffered Saline solution (PBS). A representative sample of FWB harvested from antral follicles injected with fluorescent liposomes are shown in Micrograph B. Granulosa cells flushed from liposome injected antral follicles are shown in Micrographs $C$ and $D$. The green fluorescence detection of liposome was detected in the mural granulosa cells layers and the major signal was located in the cells' plasma membrane (Insert in micrograph B), which is also confirmed in Micrographs $C$ and D. Nuclei are counterstained in blue with DAPI. 